\title{
Unilateral condylar hyperplasia recurrence after orthognathic surgery: a case report
}

\author{
Follacchio GA ${ }^{1}$, Ricci $M^{1}$, Ramieri $V^{2}$, Vellone $V^{2}$, Frantellizzi $V^{1,3 *}$, Liberatore $M^{1}$, Monteleone $F^{1}$, Civitelli L ${ }^{1}$, Cascone $P^{2}$ and De Vincentis $G^{1}$ \\ ${ }^{1}$ Department of Radiological Sciences, Oncology and Anatomical Pathology, Sapienza University of Rome, Rome, Italy \\ ${ }^{2}$ Department of Maxillo-Facial Surgery, Sapienza University of Rome, Rome, Italy \\ ${ }^{3}$ Angio-Cardio-Thoracic Pathophisiology and Imaging, "Sapienza” University of Rome, Rome, Italy
}

\begin{abstract}
Introduction: Unilateral Condylar Hyperplasia (UCH) is an uncommon condition resulting in facial asymmetry and malocclusion. At the time of diagnosis, an accurate evaluation of condylar activity through bone SPECT is necessary to determine the most appropriate surgical treatment. The Authors present a case of a UCH recurrence after orthognathic surgery in order to discuss about the clinical role of condylar SPECT in UCH therapeutic management.

Case report: A 60-years old female patient was referred to the Department of Maxillo-Facial Surgery of Sapienza University of Rome for a relapse of mandibular laterodeviation. At the age of 24 , she underwent orthognathic surgery after a diagnosis of UCH without condylar SPECT evaluation.

As part of our clinical routine, condylar SPECT was performed and a significant difference in radiotracer uptake (20\%) was found between the left condylar region and the contralateral one. She underwent high condilectomy and Bilateral Mandibular Sagittal Osteotomy. She had no evidence of recurrence till today.

Discussion: Optimal surgical management of UCH is still controversial. In the diagnostic phase of $\mathrm{UCH}$, a functional evaluation of condylar growth status by bone SPECT is an essential step to avoid long-term recurrence of mandibular asymmetry. On the basis of condylar metabolic activity, UCH can be classified in an active phase or a stationary phase. Clinical and functional evaluation of patients with mandibular asymmetry should be standardized in order to plan the most appropriate surgical and orthodontic treatment.
\end{abstract}

\section{Introduction}

Unilateral Condylar Hyperplasia (UCH) is an uncommon disorder characterized by an independent and excessive growth of one mandibular condyle as compared to the contralateral one. $\mathrm{UCH}$ pathogenesis is still unknown. Suggested etiologies include endocrine disorders, metabolic hyperactivity, trauma, arthrosis and genetics [12]. UCH usually develops in the growth phase, nevertheless rare cases in adult patients are reported [3]. Although UCH is considered a selflimiting condition, progressive condylar growth can result in both esthetic and functional problems, such as facial asymmetry, occlusal alterations and Temporo-Mandibular Joint (TMJ) dysfunction. Differential diagnosis for UCH includes maxillary hypoplasia, mandibular prognathism, dislocation of the condyle anterior to the articular eminence, acromegaly, osteochondroma and contralateral condylar resorption [4].

Diagnosis of UCH depends on an exhaustive anamnesis and clinical examination associated to an accurate imaging study. In this specific clinical setting, the diagnostic gold standard is represented by clinical diagnosis [5].

On the one hand, diagnostic imaging such as teleradiography, orthopantomography and cone beam CT scan are used to quantify the grade of hyperplasia and represent the basis for surgical planning.

On the other hand, nuclear imaging study is essential to assess the state of metabolic activity of the condylar head. Bone scintigraphy with ${ }^{99 \mathrm{~m}} \mathrm{Tc}$-Diphosphonates acquired with Single Photon Emission Computed Tomography (SPECT) technique allows a quantitative analysis of bone metabolic activity in the hyperplastic condyle compared to the contralateral one. After clinical diagnosis, the accurate assessment of condylar growth state is of primary importance to determine a correct therapeutic management. The activity level of the condyle is, in fact, strongly correlated with mandibular asymmetry and the risk of recurrence [6,7].

$\mathrm{UCH}$ treatment is primarily surgical and often associated to orthodontics to correct malocclusion.

Patients with a stable hyperplastic process, as assessed by bone SPECT, can be treated with orthodontics and orthognathic surgery. Otherwise, for patients with an active UCH different treatment options are currently reported by literature: condylectomy of the hyperplastic condyle, condylectomy associated to orthognatic surgery, or orthognatic surgery alone.

The Authors report the case of a female patient referred to the Department of Maxillo-Facial Surgery of Sapienza University of Rome at the age of 51 for mandibular laterodeviation. At the age of 24 she was diagnosed left UCH and treated with orthognathic surgery, without previous bone SPECT evaluation of condylar growth state. 27 years

Correspondence to: Dr. Viviana Frantellizzi, Viale Regina Elena,324, 00161 Rome, Italy, Tel: 0649978596; Fax: 0649978592; E-mail: viviana.frantellizzi@ uniroma1.it

Key words: Unilateral Condylar Hyperplasia - bone SPECT - high condilectomy Received: July 19, 2017; Accepted: August 14, 2017; Published: August 17, 2017 
later, she presented a relapse of mandibular asymmetry. The Authors' aim was to discuss the clinical and prognostic role of bone SPECT and its impact on surgical management in patients with Unilateral Condylar Hyperplasia.

\section{Case report}

The patient is a 60-years old female. During the growth phase, she developed a mandibular laterodeviation. She was diagnosed with left $\mathrm{UCH}$ and at the age of 24 she underwent orthognatic surgery consisting in Le Fort I osteotomy associated to Bilateral Sagittal Split Osteotomy (BSSO).

At the age of 51, the patient was referred to the Department of Maxillo-Facial Surgery of Sapienza University of Rome for relapse of the mandibular laterodeviation. Clinically, she presented a facial asymmetry of the lower face with mandibular midline shifted to the right and deviation in the opening to the left side. The patient complained about TMJ right pain and clicking. Bilateral temporal migraine was reported.

Physical examination showed mandibular asymmetry with an oral rim deflected to the bottom in the left side. Intraoral examination revealed an anterior open bite, occlusal canting and a mandibular intercisal line shifted $3 \mathrm{~mm}$ to the right (Figure 1).

The patient underwent orthopantomography, anteroposterior and lateral cephalograms and cone beam CT showing a significant change in the anatomy of the left mandibular condyle, which resulted grossly deformed with elongation of the left mandibular ramus (Figure 2).

After the diagnosis of left UCH recurrence, the patient started the orthodontic preparation in order to correct malocclusion before surgical treatment. Eight months later, the patient was ready for surgery and a bone SPECT scintigraphy with ${ }^{99 \mathrm{~m} T c-D i p h o s p h o n a t e s}$ was performed to evaluate left condylar growth activity. The cranial SPECT study was performed 2 hours after ${ }^{99 \mathrm{~m} T c-M e t h y l e n e-D i p h o s p h o n a t e}$ (MDP) intravenous injection. SPECT images were acquired with LEHR collimator, $128 \times 128$ matrix, $360^{\circ}$ orbit, 30 seconds per frame. Data were reconstructed into coronal, sagittal and transversal images using Butterworth low-pass filter, 0.5 cut-off frequency, Chang's attenuation correction threshold 12. Coronal images of the skull were displayed and ROIs were drawn on a subset of three consecutive and most representative slices displaying condylar regions, on both left and right side (Figure 3).

The difference in isotope uptake between the affected condyle and the contralateral was assessed by applying the following formula:

Percentage ratio of isotope uptake $(\Delta \%)=\frac{\text { uptake in affected side }(A)-\text { uptake in unaffected site }(B)}{A+B} \times 100$ assuming as pathological threshold a value greater than $7 \%$ [8].

Quantitative analysis of ROIs assessed that there was a significant difference in tracer uptake between the left condylar region and the contralateral side, resulting in a difference ratio of $20 \%$.

On the basis of the metabolic condylar state, the surgical approach consisted in high condylectomy with simultaneous BSSO in order to correct malocclusion.

Under general anesthesia, the surgical procedure was performed using a minimally invasive left preauricular pretragic access. By blunt dissection, after the exposure of the deep temporal fascia and ligation of superficial temporal vessels the TMJ was exposed. The TMJ lateral ligament was incised due to enter in the inferior TMJ compartment.

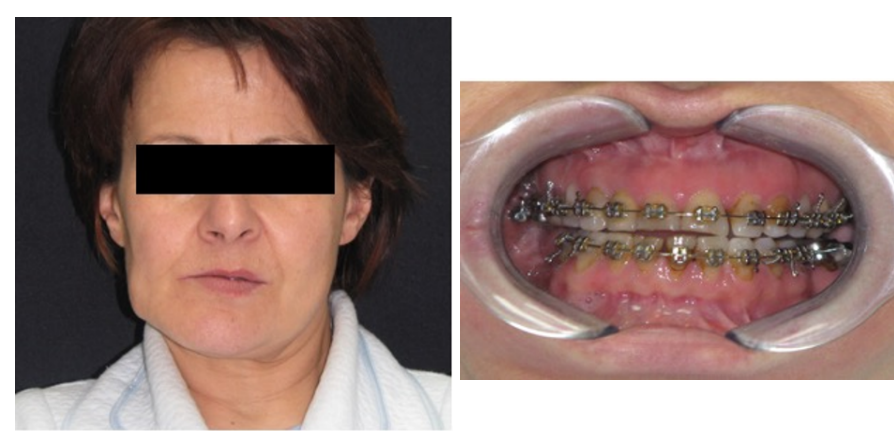

Figure 1. Clinical characteristics of the patient after UCH relapse, showing facial and occlusal deviation to the non-affected side
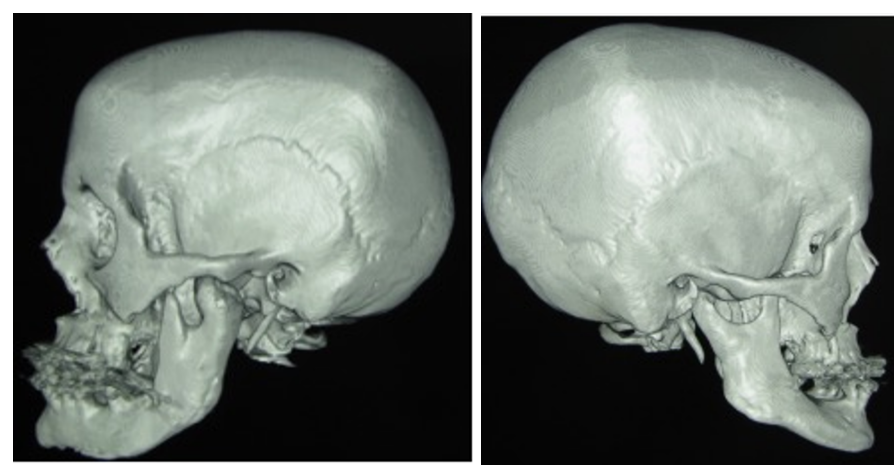

Figure 2. Pre-operative cone beam CT: 3D reconstruction showing the abnormal volume of the left condyle

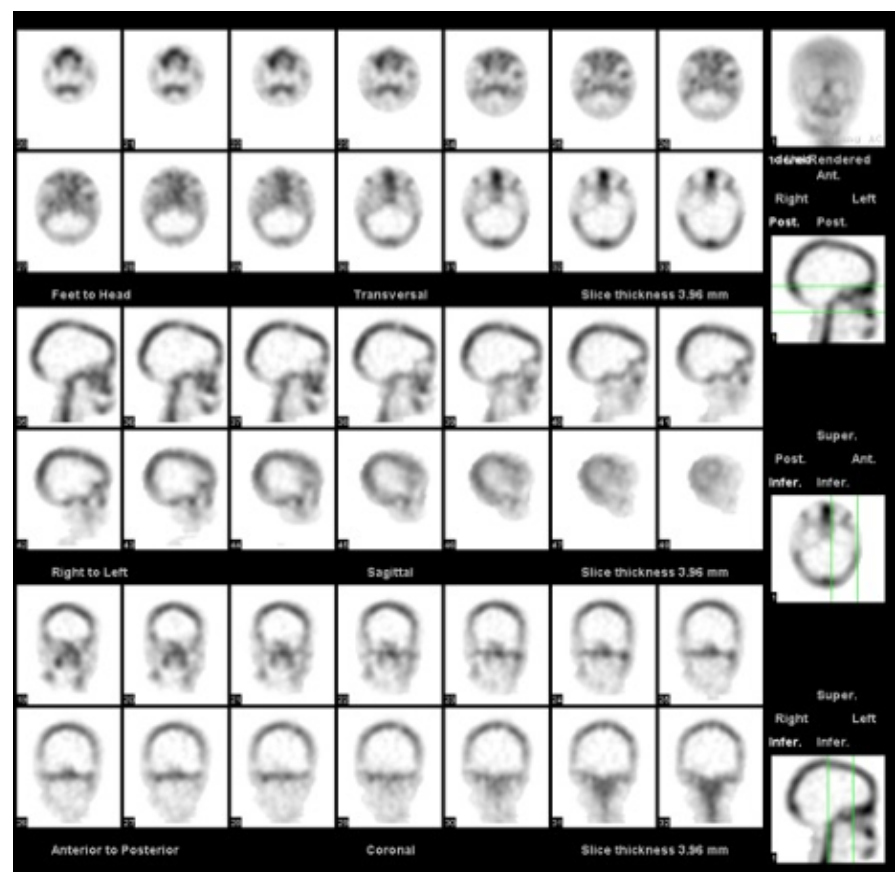

Figure 3. Condylar SPECT scan performed after UCH relapse, showing abnormal hyperactivity in the left condyle, observed in the axial, sagittal and coronal reconstructions

The condylar head was exposed subperiosteally and a proportional condylectomy of $9 \mathrm{~mm}$ was performed with Piezo Surgery. The articular disk was repositioned through Mitek Microfix QuickAnchor Plus 1.3. The anchor was linked to a 3.0 Ethibond permanent suture, which is used to fix the posterolateral side of the disk above the condyle. A functional test of joint mobility was then performed by opening and 
closing the jaw and moving it from side to side, checking the correct position of the disk [9].

Histopathologic examination of the specimen confirmed the clinical diagnosis of left UCH (type 1), showing a broad proliferation area and the presence of cartilage islands in the deep layers of trabecular bone of the hyperplastic condyle.

After surgery, orthodontic elastics were positioned to place the osteotomized condyle in the glenoid fossa, regardless of the contralateral open bite.

No facial nerve alterations were observed. Within 6 months, the patient reached a balanced occlusal plane and a good functional stability of the TMJ (Figure 4). The patient has been followed up for 8 years without clinical signs of recurrence.

\section{Discussion}

The optimal surgical management of $\mathrm{UCH}$ is still controversial. A pivotal study conducted in 2002 by Wolford et al. stated that the surgical approach based on high condylectomy associated to orthognathic surgery is the most effective treatment for patients with active $\mathrm{UCH}$, both functionally and esthetically [4]. Another study supporting the combined procedure also indicated that orthognathic surgery not supported by a direct intervention on the affected condyle could lead to future issues, because condylar growth may not be complete at the time of surgery [10]. It has been showed, that high condylectomy improves long-term outcome in UCH patients arresting the not proportionate growth of the condyle with the surgical removal of the principal mandibular growth site.

According to Fariña et al, the Authors believe that proportional condylectomy can be used as the sole surgical treatment in cases of $\mathrm{UCH}$, avoiding the need for secondary orthognathic surgery [11].

By removing the difference observed between the measurements of the hyperplastic and the healthy side, proportional condylectomy allows the centering of the interincisal lines and the lateral movement towards the affected side. It is the Authors' opinion that the restoration of the condyle-disc integrity should be preferred to a perfect symmetry of condylar heights. A correct post-surgical functioning, in fact, will provide condylar remodeling and, consequently, a proper occlusion.

A correct planning of $\mathrm{UCH}$ treatment always depends on a complete clinical examination of facial asymmetry, malocclusion and TMJ dysfunctions. Radiological studies are essential to evaluate the morphologic features of the hyperplastic condyle and to prepare a personalized surgical treatment. Besides morphologic characteristics,
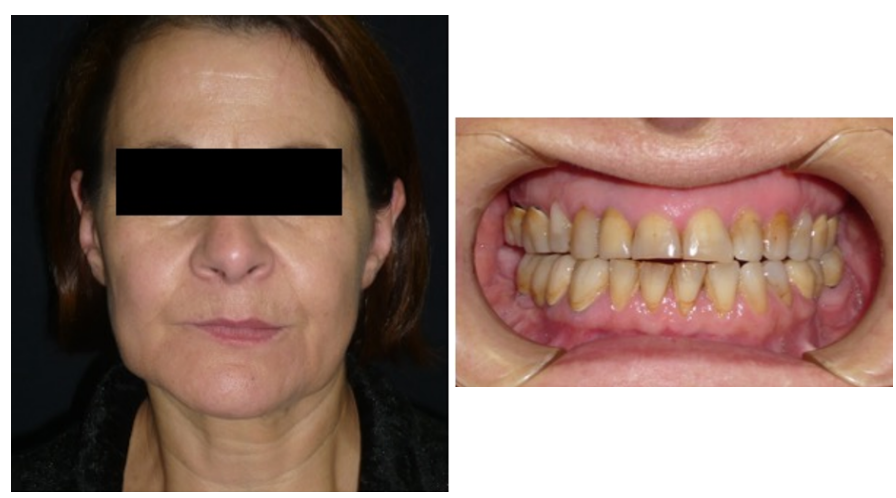

Figure 4. Clinical characteristics of the patient after high condilectomy, showing partial reduction of facial asymmetry and a balanced occlusal plane evaluation of UCH patients should always include a precise assessment of condylar growth state. On the basis of condylar metabolic activity, $\mathrm{UCH}$ can be classified in an active phase or a stationary phase. This distinction is crucial because the condylar activity status is highly correlated to the grade of mandibular asymmetry and the risk of recurrence after surgery. Bone SPECT scintigraphy is the main diagnostic tool used to assess the actual activity status of the hyperplastic condyle. This procedure is widely used in the evaluation of patient with suspected UCH. Literature based on control studies indicate that normal individuals or patients without progressive facial asymmetry show a difference in radiotracer uptake values between the left and right condylar regions less than $10 \%$, whereas a difference greater than $10 \%$ is strongly suggestive of active UCH [12]. Nevertheless, a systematic review of the literature by Saridin et al. reported a large variability between studies from different centers performing SPECT scan [5]. Besides the $10 \%$ cut-off value reported by Saridin and colleagues, it is the Authors' opinion that every Nuclear Medicine center should define their own cut-off value based on affected patients and a control group.

Based on this multi-disciplinary experience with $\mathrm{UCH}$ patients, affected side high condylectomy must be considered after the evidence of positive SPECT scan in order to contain condylar overgrowth and to prevent recurrence of facial asimmetry. Conversely, a normal SPECT scan should allow to operate a correction of occlusal and facial deformities through orthognathic surgery alone.

The reported case is a clear example of recurrence secondary to an incomplete assessment of an active UCH. Failure in recognize this condition can result in unfavorable functional and esthetic treatment results. At the time of first clinical examination, a functional evaluation of condylar growth status by the means of a bone SPECT would have informed about the need to perform a removal of the hyperplastic condylar head to prevent a long-term recurrence of mandybular laterodeviation.

\section{References}

1. Egyedi P (1969) Aetiology of condylar hyperplasia. Aust Dent J 14: 12-17. [Crossref]

2. Raijmakers PG, Karssemakers LH, Tuinzing DB (2012) Female predominance and effect of gender on unilateral condylar hyperplasia: a review and meta-analysis. J Oral Maxillofac Surg 70: e72-76. [Crossref]

3. Nitzan DW, Katsnelson A, Bermanis I, Brin I, Casap N (2008) The clinical characteristics of condylar hyperplasia: experience with 61 patients. J Oral Maxillofacial Surg 66: 312-318

4. Wolford LM, Mehra P, Reiche-Fischel O, Morales- Ryan CA, García-Morales P (2002) Efficacy of high condylectomy for management of condylar hyperplasia. Am J Orthod Dentofacial Orthop 121: 136-150

5. Saridin CP, Raijmakers PG, Tuinzing DB, Becking AG (2011) Bone scintigraphy as a diagnostic method in unilateral hyperactivity of the mandibular condyles: a review and meta-analysis of the literature. Int J Oral Maxillofac Surg 40: 11-17

6. Jones RH, Tier GA (2012) Correction of facial asymmetry as a result of unilateral condylar hyperplasia. J Oral Maxillofac Surg 70: 1413-1425. [Crossref]

7. Karssemakers LH, Raijmakers PG, Nolte JW, Tuinzing DB, Becking AG, (2013) Interobserver variation of single photon emission computed tomography bone scans in patients evaluated for unilateral condylar hyperactivity," Oral Surgery, Oral Medicine Oral Pathology, Oral Radiology 115: 399-405

8. Criscuoli B, Remediani S, Fioravanti M, Monteleone F, Massa R, et al. (2008) SPECT bone scintigraphy in unilateral condylar hyperplasia: a comparision between different views and a threshold of isotopic uptake between unaffected and affected side. Eur $J$ Nucl Med Mol Imaging 35: S152-S152

9. Spallaccia F, Rivaroli A, Basile E, Cascone P (2013) Disk repositioning surgery of the temporomandibular joint with bioabsorbable anchor. J Craniofac Surg 24: 1792-1795. [Crossref]

10. Villanueva-Alcojol L, Monje F, Gonzalez-Garcia R, (2011) Hyperplasia of the mandibular condyle: clinical, histopathologic, and treatment considerations in a series of 36 patients. J Oral Maxillo-facial Surg 69: 447-455 
Follacchio GA (2017) Unilateral condylar hyperplasia recurrence after orthognathic surgery: a case report

11. Fariña R, Olate S, Raposo A, Araya I, Alister JP, et al. (2016) High condylectomy versus proportional condylectomy: is secondary orthognathic surgery necessary? Int $J$ Oral Maxillofac Surg 45: 72-77. [Crossref]
12. Pripatnanont P, Vittayakittipong P, Markmanee U, Thongmak S, Yipintsol T (2005) The use of SPECT to evaluate growth cessation of the mandible in unilateral condylar hiperplasia. Int J Oral Maxillofac Surg 34: 364-368

Copyright: $@ 2017$ Follacchio GA. This is an open-access article distributed under the terms of the Creative Commons Attribution License, which permits unrestricted use, distribution, and reproduction in any medium, provided the original author and source are credited. 